\title{
Dynamic Influences on Coincidence Detection in Neocortical Pyramidal Neurons
}

\author{
Lucinda A. Grande, ${ }^{3}$ Gregory A. Kinney, ${ }^{2}$ Greta L. Miracle, ${ }^{3}$ and William J. Spain ${ }^{1,3}$ \\ Departments of ${ }^{1}$ Physiology and Biophysics, Neurology, and ${ }^{2}$ Rehabilitation Medicine, University of Washington, Seattle, Washington 98105 , and ${ }^{3}$ Veterans \\ Affairs Puget Sound Health Care System, Seattle, Washington 98108
}

\begin{abstract}
The firing rate of neocortical pyramidal neurons is believed to represent primarily the average arrival rate of synaptic inputs; however, it has also been found to vary somewhat depending on the degree of synchrony among synaptic inputs. We investigated the ability of pyramidal neurons to perform coincidence detection, that is, to represent input timing in their firing rate, and explored some factors that influence that representation. We injected computer-generated simulated synaptic inputs into pyramidal neurons during whole-cell recordings, systematically altering the phase delay between two groups of periodic simulated input events. We explored how input intensity, the synaptic time course, inhibitory synaptic conductance, and input jitter influenced the firing rate representation of input timing. In agreement with computer modeling studies, we found that input synchronization increases firing rate when intensity is low but reduces firing rate when intensity is high. At high intensity, the effect of synchrony on firing rate could be switched from reducing to increasing firing rate by shortening the simulated excitatory synaptic time course, adding inhibition (using the dynamic clamp technique), or introducing a small input jitter. These opposite effects of synchrony may serve different computational functions: as a means of increasing firing rate it may be useful for efficient recruitment or for computing a continuous parameter, whereas as a means of decreasing firing rate it may provide gain control, which would allow redundant or excessive input to be ignored. Modulation of dynamic input properties may allow neurons to perform different operations depending on the task at hand.
\end{abstract}

Key words: coincidence detection; synchrony; phase delay; neocortex; pyramidal neuron; synaptic inhibition; jitter; dynamic clamp

\section{Introduction}

Coincidence detection (CD) is the operation performed by a neuron when it responds maximally to synchronized inputs. Various neuronal processes may be modulated as a function of the timing of synaptic inputs, such as intracellular calcium concentration or output spike train features. One feature of the output spike train of a neuron that may depend on input timing is firing rate. For firing rate to be an effective $\mathrm{CD}$ signal it must represent the relative timing of inputs both sensitively and reliably.

Coincidence detectors in the auditory brainstem use firing rate to signal input timing to help identify the location of a sound (Goldberg and Brown, 1969; Carr and Konishi, 1990). These highly specialized neurons compute interaural time difference using the phase delay between two groups of periodic synaptic inputs, each group encoding sound waves arriving at one ear. In neocortex, the use of input timing for computation is an appealing theory that would allow efficient information representation and transfer (Abeles, 1991; Rieke et al., 1996). A role has been proposed for input phase delay in pattern recognition tasks such

Received July 26, 2003; revised Dec. 5, 2003; accepted Dec. 30, 2003.

This work was supported by a Veterans Affairs Merit Review. We thank Eberhard Fetz, Fred Rieke, and Peter Schwindt for helpful comments on this manuscript, Mark Binder for helpful discussions, Hans van Brederode for assistance with the experiments, and Richard Lee for technical assistance.

Correspondence should be addressed to William J. Spain, Veterans Affairs Puget Sound Health Care System, 1660 South Columbian Way, Seattle, WA 98108. E-mail: spain@u.washington.edu.

DOI:10.1523/JNEUROSCI.3500-03.2004

Copyright $\odot 2004$ Society for Neuroscience $\quad$ 0270-6474/04/241839-13\$15.00/0 as sensory perception (Hopfield, 1995; Gerstner et al., 1997). Although some studies have suggested the potential for neocortical neurons to use input timing for computation (Mainen and Sejnowski, 1995; Harsch and Robinson, 2000), their capabilities remain poorly understood.

Pyramidal neurons, the primary relay and output neurons of the cerebral cortex, are clearly not specialized for CD. Sensitivity would be limited by their long membrane time constant, which would result in temporal summation of many synaptic input currents over a typical interspike interval of 25-100 msec (Shadlen and Movshon, 1999; but see Koch et al., 1996). Reliability would be limited because they fire repetitively, increasing their firing rate in response to increases in input current intensity (Schwindt et al., 1997).

Experimental and computer modeling studies, however, predict that the firing rate of pyramidal neurons would be influenced somewhat by input timing (Segundo et al., 1963; Reyes and Fetz, 1993; Bernander and Koch, 1994; Murthy and Fetz, 1994; Mainen and Sejnowski, 1995; Marsalek et al., 1997; Rudolph and Destexhe, 2001; Oviedo and Reyes, 2002). According to Bernander and Koch (1994) and Murthy and Fetz (1994), a high degree of synchrony among inputs could either increase or decrease firing rate, depending on input intensity. This complex response to both the timing and intensity of inputs would seem to confound interpretation of firing rate. Optimal use of firing rate to signal either of these input features would require that the influence of the other be suppressed. 
We investigated the ability of neocortical pyramidal neurons to perform one type of $\mathrm{CD}$ by exploring their firing rate representation of input phase delay. We injected computer-generated current trains into pyramidal neurons during whole-cell recordings, systematically altering the phase delay between two groups of periodic simulated input events. We first explored the interaction between the influences of input phase delay and intensity on firing rate. We then analyzed the effectiveness of two potential mechanisms for reducing the influence of input intensity: short synaptic time constants and inhibitory synaptic conductance. We also investigated the role of variation in the arrival time of input events (jitter) within each group, an important constraint on CD in pyramidal neurons.

\section{Materials and Methods}

Slice preparation. Postnatal day 14-28 Sprague Dawley rats were deeply anesthetized in a sevoflurane-filled chamber and quickly decapitated. A coronal hemisection $(\sim 0.5 \mathrm{~cm})$ of somatosensory cortex was removed with a razor blade and submerged in a cooled $\left(\sim 4^{\circ} \mathrm{C}\right)$ bath of cutting solution in the chamber of a Vibratome tissue slicer (TPI, St. Louis, MO). Cutting solution contained (in $\mathrm{mm}$ ): $5 \mathrm{KCl}, 1.25 \mathrm{KH}_{2} \mathrm{PO}_{4}, 26 \mathrm{NaHCO}_{3}, 5$ $\mathrm{MgCl}_{2}, 20$ TEA-Cl, 105 choline-Cl, 20 sucrose, 10 dextrose, 1.3 ascorbic acid, and 2.4 pyruvic acid (320 mOsm) gassed with $95 \% \mathrm{O}_{2} / 5 \% \mathrm{CO}_{2}$ to maintain $\mathrm{pH}$ at 7.4. Coronal slices $(300 \mu \mathrm{m}$ thick) were cut and transferred to a holding chamber filled with artificial CSF (ACSF) containing (in mM): $130 \mathrm{NaCl}, 26 \mathrm{NaHCO}_{3}, 3 \mathrm{KCl}, 2 \mathrm{CaCl}_{2}, 2 \mathrm{MgCl}_{2}, 1.25 \mathrm{NaH}_{2} \mathrm{PO}_{4}$, and 10 dextrose, gassed with $95 \% \mathrm{O}_{2} / 5 \% \mathrm{CO}_{2}$. The temperature of the holding chamber was maintained at $35^{\circ} \mathrm{C}$ for $1 \mathrm{hr}$ and was then allowed to cool to room temperature.

Whole-cell recordings. Individual slices were transferred to a $0.5 \mathrm{~cm}^{3}$ recording chamber mounted on an upright Zeiss microscope (Thornwood, NY) with fixed stage and perfused at a rate of $\sim 1.5 \mathrm{ml} / \mathrm{min}$ with warmed $\left(31-35^{\circ} \mathrm{C}\right.$, average $\left.32.4 \pm 0.6\right)$ ACSF gassed with $95 \% \mathrm{O}_{2} / 5 \%$ $\mathrm{CO}_{2}$. Layer $\mathrm{V}$ pyramidal neurons were visually identified on a monitor using a $40 \times$ water-immersion lens with differential interference contrast optics illuminated with near-infrared light $(750-800 \mathrm{~nm})$.

Electrodes were drawn from $75 \mu$ l borosilicate hematocrit tubing (VWR, San Francisco, CA) coated with Sylgard 184 (silicone elastomer kit, Dow Corning, Midland, MI) for capacitance reduction and filled with (in mM): 0.1 EGTA, $5 \mathrm{KCl}, 0.5 \mathrm{Na}-\mathrm{GTP}$ (Sigma, St. Louis, MO), 2 $\mathrm{MgCl}_{2}, 10 \mathrm{HEPES}, 135 \mathrm{KCH}_{3} \mathrm{SO}_{4}$, and $2 \mathrm{Na}_{2}$-ATP. Values given for voltages were corrected for a measured liquid junction potential of 7.5 $\mathrm{mV}$.

Experiments were performed with an Axoclamp 2A (Axon Instruments, Foster City, CA) amplifier in bridge mode. Electrode resistance and capacitative current were checked periodically and nulled using the Axoclamp 2A circuitry. Excitatory inputs were applied in current-clamp mode to represent synaptic currents arriving at the soma after dendritic integration. Inhibitory synaptic conductance was applied using the dynamic clamp technique (Robinson and Kawai, 1993; Sharp et al., 1993). This was intended to simulate the dynamic alterations in current presumed to occur because of activity at $\mathrm{GABA}_{\mathrm{A}}$ receptor-mediated synapses at the soma and proximal dendrites. In some experiments, excitatory inputs were applied in dynamic clamp mode for comparison with the current-clamp experiments. Voltage and current traces were low-pass filtered at $10 \mathrm{kHz}$ and recorded on videotapes using pulse-code modulation with a frequency response of 44 $\mathrm{kHz}$. Post hoc data analysis was done using homemade programs or by pClamp 6 (Axon Instruments).

Computer simulation of synaptic currents. Pyramidal neurons were stimulated intracellularly with computer-generated current that resembled the summation of synaptic events from two groups of inputs. Current was injected during somatic recording and thus represented postsynaptic currents (PSCs) at the soma after dendritic integration. Somatic current injection provides insight into the computation used at a final common pathway for synaptic current flow (the soma) to produce an action potential output but cannot be used to infer what types of computations might be performed upstream on dendritically distributed
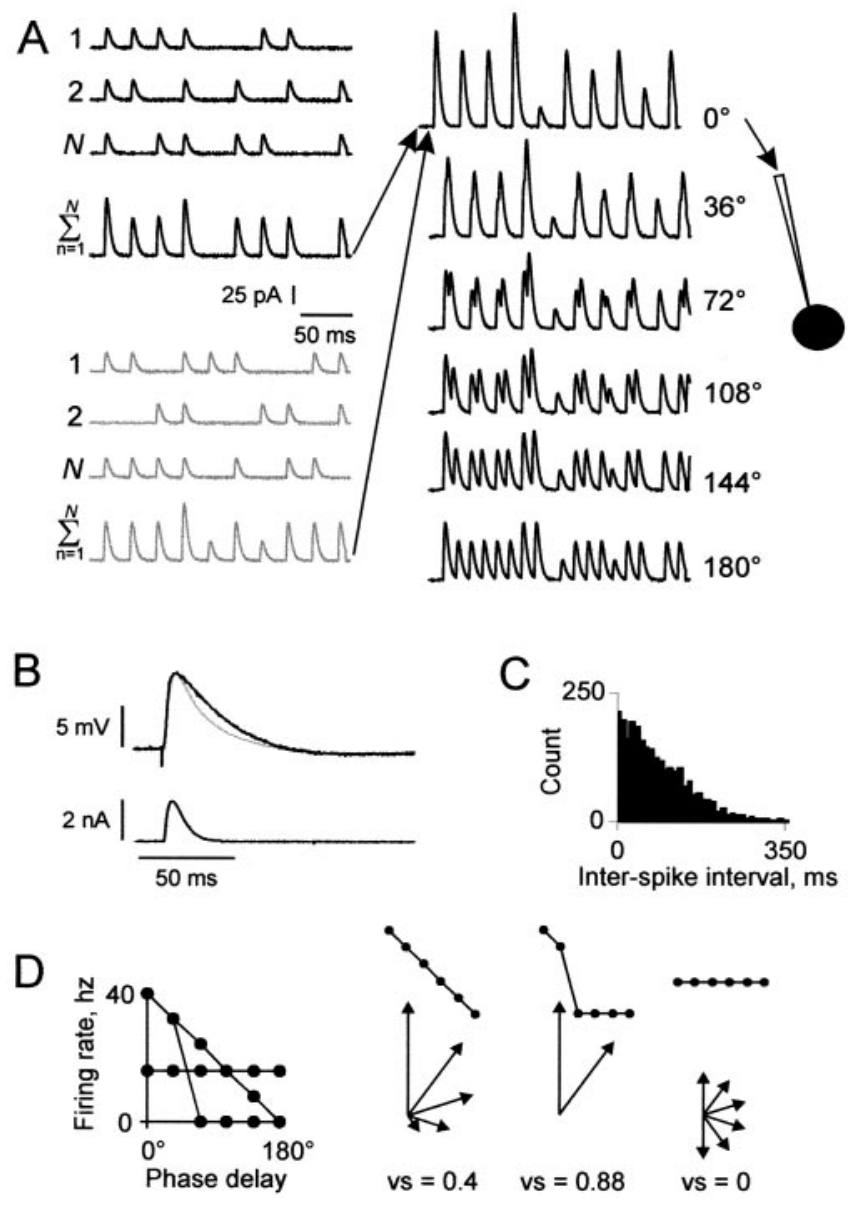

Figure 1. Method of generating input currents. A, Phase-delayed current trains. Left, Input to the recorded neuron was composed of simulated synaptic currents arriving at the soma from two input groups, each composed of $N$ inputs. In this example, the two input groups are black and gray, and $N=3$. Each of the $N$ inputs produced a 250 msec current train with an average firing rate of $25 \mathrm{~Hz}$. Simulated synaptic events in the shape of $\alpha$ waves $\left(\tau_{\text {syn }}=2 \mathrm{msec}\right.$ in this example), with peak amplitude $k=25 \mathrm{pA}$, were phase-locked to a $40 \mathrm{~Hz}$ sinusoid, with the presence or absence of an event during each cycle determined probabilistically. Jitter was introduced by applying to the synaptic event time a random offset relative to the peak of the sinusoid and was specified as the SD, $\sigma$, of the random distribution ( $\sigma=0 \mathrm{msec}$ in this example). Current trains from all inputs within a group were summed. Center, The onset of one group's train was delayed before summation with the other group's train, with the onset delay increased systematically during each of six runs in increments of $2.5 \mathrm{msec}$ (representing phase delays of $0^{\circ}$ through $180^{\circ}$ of a $40 \mathrm{~Hz}$ cycle). Right, Each of the combined current trains was injected into the recorded neuron (black circle) in whole-cell mode. $B$, Comparison of the time course of an evoked and simulated PSP. A PSP evoked by extracellular stimulation (black trace) is overlaid with a simulated PSP (gray trace) evoked by injection of a simulated PSC (bottom trace) generated using Equation 1 with $\alpha=0.25$ ( $\tau_{\text {syn }}=4 \mathrm{msec}$ ). In 17 cells in which a PSP was evoked by extracellular stimulation, the range of $\tau_{\text {syn }}$ that evoked simulated PCPs of similar time course was $1.7-5 \mathrm{msec}$ (mean $\pm S D=2.5 \pm 0.9$ ). C, Typical distribution of interevent intervals used for simulated inhibitory synaptic inputs ( $\sigma=25 \mathrm{msec}$ ). D, Graphic depiction of PD index calculation. Left, Plot of three hypothetical PD functions. Right, Each PD function in the plot on the left is reshown above the corresponding vector representations of data points.

synaptic inputs before flow into the soma. A computer program, written in the C programming language for a Macintosh Quadra 950 and adapted from Reyes et al. (1996), calculated the net synaptic current at the soma and injected the current into the neuron via the amplifier and electrode. Excitatory inputs were described by three parameters that were varied independently: (1) the number of excitatory inputs $(N)$ to the recorded pyramidal neuron, $(2)$ the time constant $\left(\tau_{\text {syn }}\right)$ of a synaptic event, specified by an $\alpha$ function (Eq. 1), and (3) jitter, specified by the SD 
$(\sigma)$ of synaptic event times relative to the start of the stimulus cycle. In addition, the average amplitude of a randomly varying inhibitory conductance could be specified.

Figure $1 A$ is a schematic of the elements of a 250 msec current train. The average firing rate of each input was $25 \mathrm{~Hz}$. Synaptic input events were phase-locked to a $40 \mathrm{~Hz}$ sinusoid representing the gamma oscillations in neocortex. For each of $N$ inputs, the presence or absence of an event during each of 10 stimulus cycles was determined randomly with a uniform distribution using a probability $(p)$ of $0.625(25 / 40 \mathrm{~Hz})$. Thus $N p$ events occurred during an average stimulus cycle.

A waveform describing the excitatory current, $I$, was generated for each event, described by the following function:

$$
I(t)=k \alpha t^{\star} \exp (1-\alpha t),
$$

where $t$ is the time, $k$ is the peak amplitude of the unitary current (fixed at $25 \mathrm{pA}$ ), and $\alpha$ is inversely related to the time course of each synaptic event. Values are given below in terms of the synaptic time constant $\left(\tau_{\text {syn }}\right)$, where $\tau_{\text {syn }}=1 / \alpha$. The range of $\tau_{\text {syn }}$ was chosen to produce simulated postsynaptic potentials (PSPs) both longer and shorter than those evoked by extracellular stimulation (Fig. 1 B) (also see Fig. 4). The control value of $\tau_{\text {syn }}(2 \mathrm{msec})$ produced a PSP that was too narrow to sustain multiple action potentials except with extremely high intensity input. In some experiments the excitatory inputs were applied using the dynamic clamp technique, with reversal potential set at $0 \mathrm{mV}$ and $g(t)$ for each synaptic event set as for $I(t)$ using Equation 1, with $k$ representing peak synaptic conductance.

We introduced jitter by systematically increasing dispersion in the timing of the periodic events. The Gaussian function was used to apply a random offset to each event time relative to the start of the cycle. We used four degrees of variation, described by SDs of 0, 3, 6, and 9 msec (see Fig. $8 C)$.

The event wave-forms generated for all inputs were summed. To represent varying phase delays between two groups of inputs, two complete 250 msec current trains were constructed, and the onset of one train was delayed before summation with the other train (Fig. $1 \mathrm{~A}$ ). The onset delay was increased systematically during each of six runs in increments of 2.5 msec. The six runs thus represented six phase delays of $0^{\circ}$ through $180^{\circ}$ of a $40 \mathrm{~Hz}$ cycle.

Finally, in the experiments in which inhibitory conductance was added, the dynamic clamp technique was used. A $250 \mathrm{msec}$ waveform was generated to represent inhibitory conductance using Equation 1, with $g_{\text {inh }}(t)$ substituted for $I(t)$ and $k$ representing peak conductance amplitude, with parameters chosen to produce a randomly fluctuating waveform. Unitary events occurred at $40 \mathrm{~Hz}$ with $p=0.625$, with a jitter level sufficient to produce random interevent intervals $(\sigma=9$ initial experiments or $25 \mathrm{msec}$ ) (Fig. 1C) (later experiments). Inhibitory intensity was adjusted by altering $N$ or $k$, with $\tau_{\text {syn }}$ fixed at $4 \mathrm{msec}$. Average inhibitory conductance $\left(G_{\text {inh }}\right)$ is given in nanosiemens. The conductance waveform was converted into current to be injected into the recorded neuron according to the following formula:

$$
I\left(V_{\mathrm{m}}, t\right)=g_{\text {inh }}(t) *\left(V_{\mathrm{m}}(t)-E_{\mathrm{rev}}\right),
$$

where $g_{\text {inh }}(t)$ is the conductance at time $t, V_{\mathrm{m}}(t)$ is the membrane potential at time $t$, and $E_{\text {rev }}$ is the reversal potential for the $\mathrm{GABA}_{\mathrm{A}}$ receptormediated conductance, set at $-66 \mathrm{mV}$ (Stuart, 1999).

At each sampling point, after injection of the excitatory current, the computer performed a $20-40 \mathrm{kHz}$ alternation of analog-to-digital (A/D) conversion and digital-to-analog (D/A) conversion: the computer sampled $V_{\mathrm{m}}(t)$, calculated Equation 2, and injected the resulting current into the neuron. Thus, the injected current varied dynamically with $V_{\mathrm{m}}$ (Robinson and Kawai, 1993; Sharp et al., 1993).

Recording protocol. The stimulus trains were injected with a pause of $3-4 \mathrm{sec}$ between trains. For each combination of input parameters, a set of six phase-delay trains was injected in sequence, and then the sequence was repeated in three to five trials, with unique trains constructed for each of the trials. An action potential was counted each time $V_{\mathrm{m}}$ exceeded a threshold (usually $-1 \mathrm{mV}$ ). The average firing rate of the recorded neuron in response to a particular phase delay, in Hertz, was calculated by multiplying by 4 the number of action potentials occurring within a 250 msec train, averaged over all trials.

This experimental design included a measurement bias, in that the start of the $250 \mathrm{msec}$ combined train lacked the contribution from the second (delayed) input group, up to $12.5 \mathrm{msec}$ at $180^{\circ}$ (5\% of the train length). This small bias was judged insignificant after comparing results obtained with the full $250 \mathrm{msec}$ versus the last $237.5 \mathrm{msec}$ in several representative neurons.

Data analysis. The plot of firing rate as a function of phase delay for a given phase-delay sequence is denoted the "phase-delay function" (PD function) (see Fig. $2 B$ ). To describe quantitatively the relationship between phase delay and firing rate, we calculated a performance measure called the "phase-delay index" (PD index) for each PD function using a method adapted from the vector strength of Goldberg and Brown (1969). $\mathrm{PD}$ index quantified the shape of the PD function with values between -1 and 1. Quantification allowed comparison of firing behavior between values of each parameter (input intensity, $\tau_{\text {syn }}, G_{\text {inh }}$, and jitter). The term "index" refers to a continuum of shapes with different qualities, not a scale from best to worst. With a PD index of 1 , all action potentials occurred at one phase delay $\left(0^{\circ}\right)$; with a PD index of 0 , action potentials were distributed evenly across phase delays, i.e., firing rate was a constant function of phase delay. PD functions with negative and positive slopes are distinguished by positive and negative PD index values, respectively. Thus a positive, zero, or negative PD index indicates that greater synchrony between the two groups increased, had no effect on, or decreased firing rate, respectively.

To calculate the PD index (Fig. 1D), each phase delay of the sequence was converted to a vector, with the magnitude given by the firing rate, and the direction calculated as the angle:

$$
\theta=\phi \cdot 2 \pi / 360
$$

where $\phi$ is the phase delay $\left(0^{\circ}\right.$ through $\left.180^{\circ}\right)$. Vectors for phase delays between $180^{\circ}$ and $360^{\circ}$ were assigned magnitudes symmetrical to those between $0^{\circ}$ and $180^{\circ}$. The vectors were summed, and the magnitude of the resulting vector, known as the vector strength, was used for the PD index. For PD functions in which firing rate was greater at $180^{\circ}$ than at $0^{\circ}$, the function curve was first flipped about its horizontal axis before vector magnitudes were assigned; the resulting vector strength was then multiplied by -1 .

Many potential measures would have adequately reflected trends in firing behavior, because, as shown in Results, firing rate was a monotonically decreasing, constant, or monotonically increasing function of phase delay. PD index reflected the shape of the PD function sensitively and with low distortion, as illustrated in Figure $2 C$, in which PD functions grouped by PD index have similar shapes. Although each PD function has a unique PD index, the reverse is not true.

Input intensity. The measure of excitatory input intensity $(E)$ was the peak current of one input group during an average stimulus cycle, normalized between neurons. It was calculated as:

$$
E=k N p / T
$$

with $k, N$, and $p$ as described above. $T$ is the current required for the neuron to reach action-potential threshold, defined as the minimum amplitude 4 msec square-wave current pulse reliably evoking an action potential from rest. $T$ closely matched $[100 \pm 6 \%$ (SEM); $n=18]$ the minimal peak simulated excitatory synaptic current with $\tau_{\text {syn }}=2 \mathrm{msec}$ (Eq. 1) reliably evoking an action potential from rest.

\section{Results}

Results are on the basis of recordings of 79 layer 5 pyramidal neurons in slices of rat somatosensory cortex. Cells were rejected 
unless a seal resistance of $>1 \mathrm{G} \Omega$ could be obtained between the electrode and cell membrane before entering the whole-cell configuration. Cells were recorded only if they showed stable resting membrane potentials $(-72.7 \pm 3.8 \mathrm{mV})$, overshooting $(>0 \mathrm{mV})$ action potentials and repetitive firing in response to an intracellular depolarizing current step, with an initial period of rapid spike-frequency adaptation. Occasionally, a slight hyperpolarizing holding potential was applied to maintain a stable resting potential. Input resistance was $86.2 \pm 36 \mathrm{M} \Omega$, as determined by the steady-state response to a 300 msec hyperpolarizing current pulse of $0.2 \mathrm{nA}$. All neurons included in this study were classified as regular spiking (Connors and Gutnick, 1990).

\section{Input intensity influences the slope of the PD function}

We first investigated the effect of phase delay on firing rate under conditions of low input intensity. We then studied how this effect varied with increasing input intensity. Figure $2 A$ contrasts the effects of low and high input intensity levels on the $V_{\mathrm{m}}$ response of a typical neuron at three phase delays. With low (top) or high (bottom) input intensity, respectively, firing rate decreased or increased as a function of phase delay.

Figure $2 B$ plots the PD function (see Materials and Methods) for a neuron supplied with four input intensity levels. The PD function slope varied, undergoing systematic change with increasing input intensity, from a negative value, to zero, to a positive value. As explained below, the slope depended on the change in peak amplitude of temporally summated PSPs at successive phase delays.

At the lowest intensity shown $(E=0.5)$, the negative slope of the PD function was caused by a decrease in total PSP amplitude arising from temporal summation as the two input groups became more separated in time. When input intensity was increased to $E=0.7$, the PD function shifted up. Higher firing rates occurred because some minimally subthreshold PSPs at each phase delay were boosted above threshold. At $E=1$, the PD function was nearly constant because, although the tail was pulled up (from 24 to $44 \mathrm{~Hz}$ at $180^{\circ}$ ), the start remained fixed $\left(40 \mathrm{~Hz}\right.$ at $0^{\circ}$ ). A firing rate of $40 \mathrm{~Hz}$ was maximal at $0^{\circ}$, because an action potential was produced during every $40 \mathrm{~Hz}$ stimulus cycle and PSPs were too narrow to sustain multiple action potentials. At $E=1.5$, the slope of the $\mathrm{PD}$ function was positive, because both the $0^{\circ}$ and $180^{\circ}$ firing rates were at their respective maxima. As seen in Figure $2 \mathrm{~A}$ (bottom), the maximal firing rate at $180^{\circ}(80 \mathrm{~Hz})$ was twice that of $0^{\circ}(40 \mathrm{~Hz})$ because PSPs occurred twice as often at $180^{\circ}$.

PD index (see Materials and Methods) was effective in demonstrating trends in firing behavior (Fig. 2C). The increasing slope of the PD function (Fig. $2 B$ ) is plotted as a steady decrease in $\mathrm{PD}$ index in Figure $2 D$. The neuron of Figure $2 B$ is represented by large symbols. Low $(E=0.5$ and 0.7$)$, medium $(E=1.0)$, and high $(E=1.5)$ intensities, at which PD function slopes are negative, zero, and positive, respectively, are plotted as positive, zero, and negative PD index values. Data points for 51 other neurons (Fig. 2D, small open circles) are clustered in a negatively sloped band showing the same trend (illustrated by the trend line).

In Figure $2 D$ and other plots of PD index versus input intensity, the axes highlight important boundaries. The horizontal axis, $\mathrm{PD}$ index $=0$, represents the boundary between positively and negatively sloped PD functions. The vertical axis, $E=1$, represents the intensity at which this boundary would be expected. Here is why. At $E=1$, the peak current of an average stimulus cycle is $k N p=T$ (Equation 4 ), which is the current required to reach action potential threshold under control conditions $\left(\tau_{\text {syn }}=2 \mathrm{msec} ; G_{\text {inh }}=0 \mathrm{nS}\right)$. At $0^{\circ}$ phase delay, current peaks of the two trains occur simultaneously, so the peak of each $40 \mathrm{~Hz}$ cycle is nearly certain to exceed $T$. In contrast, at $180^{\circ}$ phase delay, current peaks of the two trains occur independently, and only half of the $80 \mathrm{~Hz}$ cycles have peak current exceeding the average, $T$. Thus a firing rate of $40 \mathrm{~Hz}$ would be expected at both $0^{\circ}$ and $180^{\circ}$ phase delays, resulting in a constant $\mathrm{PD}$ function. In Figure $2 D$, the trend line passes just above and to the right of the intersection of the axes, indicating that a slightly higher than expected intensity was required to reach threshold during an average stimulus cycle. This was most likely attributable to intrinsic membrane conductances that produced net hyperpolarization during the course of each train.

The negative slope of the PD index trend line shows that as input intensity was increased, synchrony between the two input groups became progressively less efficient in increasing the firing rate of the neuron, until it eventually began to dampen firing rate. The opposing effects of input synchrony on firing rate indicate unreliable $\mathrm{CD}$, i.e., firing rate could not reliably provide information to a target neuron about input phase delay. If firing rate is to 
A

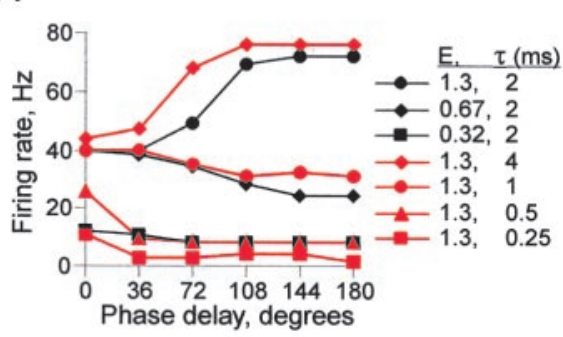

C

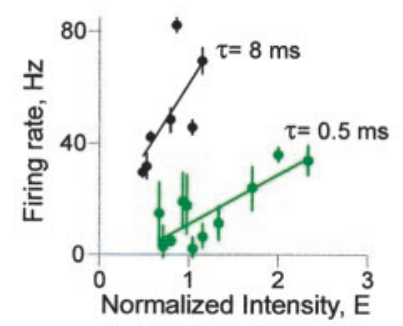

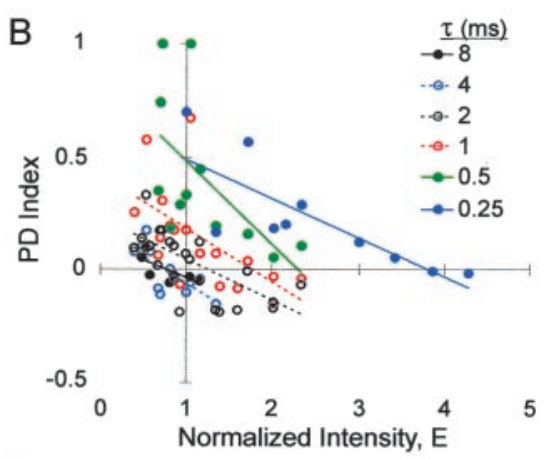

D

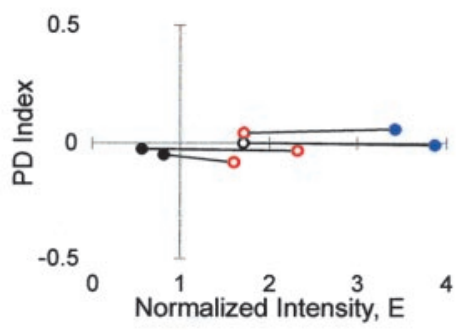

Figure 3. The effects of synaptic time course $\left(\tau_{\text {syn }}\right)$ on $C D$. $A$, Firing rate versus phase delay comparing the control value of $\tau_{\text {syn }}$ at three input intensities ( 2 msec, black symbols) with different values of $\tau_{\text {syn }}$ at one input intensity (red symbols). B, PD index versus input intensity at different values of $\tau_{\text {syn }} ; n=12$ neurons. C, Average firing rate versus input intensity for all PD functions at two values of $\tau_{\text {syn }} ; n=12$ neurons. Each data point represents the average of firing rates at all six phase delays for one PD function; error bars show SD. D, Four examples in which both the slope and $Y$-intercept of the PD function were maintained by shortening $\tau_{\text {syn }}$ as intensity was increased. For the two PD functions within a neuron (connected by lines), both PD index and firing rate (average of all 6 phase delays) differed by $<10 \%$. The symbols for $\tau_{\text {syn }}$ are the same as in $B$.

be used as a signaling code for input phase delay, one or more devices must be available to improve CD reliability. In the remainder of our study we explored the effectiveness of several mechanisms that might potentially reduce the influence of input intensity on firing rate.

\section{The synaptic time constant $\left(\tau_{\text {syn }}\right)$ alters the slope and $Y$-intercept of the PD function}

Variation in membrane conductance may be a physiologically realistic mechanism for compensating for the effect of input intensity on the PD function. In a modeling study, Bernander et al. (1991) showed how an increase in membrane conductance (attributable to increased synaptic noise), by shortening the membrane time constant, would reduce PSP time course and consequently reduce temporal summation between asynchronous inputs [see also Softky (1994) and Ho and Destexhe (2000)]. Koch et al. (1996) suggested that the membrane time constant varies dynamically depending on global network activity level. In an in vivo study, Destexhe and Pare (1999) demonstrated the physiological significance of this effect by finding a fivefold mean increase in membrane conductance during periods of high synaptic activity compared with the resting state.

Recent studies have shown that there are subcellular differences in membrane conductance between dendritic and somatic compartments that affect temporal integration (Pouille and Scanziani, 2001; Oviedo and Reyes, 2002; Williams and Stuart, 2002). To simulate an alteration of membrane conductance in the dendritic compartment, we altered $\tau_{\text {syn }}$ of our input currents. Short values of $\tau_{\text {syn }}$ simulated a state of high synaptic noise, in which a short dendritic membrane time constant would result in rapid PSP rise and decay. Long values of $\tau_{\text {syn }}$ simulated a resting state, in which long-distance signal propagation would result in an extended rise and decay (Rall, 1977). Dendritic calcium spikes cause a similar elongation (Hausser et al., 2000).

In most experiments we applied excitatory inputs in current clamp rather than dynamic clamp mode, for two reasons. First, the somatic conductance change from dendritic excitatory inputs is relatively small compared with inhibitory inputs attributable to the electrotonic distance of most excitatory synapses from the soma. Second, use of current clamp allowed us to maintain a constant simulated PSP time course while we explored PD index as a function of input intensity for each $\tau_{\text {syn }}$ (Fig. $3 B$ ). In contrast, in dynamic clamp mode, each increase in input intensity would have been accompanied by an increase in conductance, which would have resulted in shorter PSPs (although PSC width remained constant), thereby altering both intensity and membrane time constant at once. For comparison, however, we did apply excitatory inputs in dynamic clamp mode in 10 cells (Fig. 4D).

We found that a shorter $\tau_{\text {syn }}$ (i.e., greater synaptic noise) could compensate for an increase in input intensity, as predicted. Figure $3 A$ illustrates how $\tau_{\text {syn }}$ affected the PD function in a neuron supplied with high intensity input $(E=1.3)$. With the control value of $\tau_{\text {syn }}$ (2 msec; black circles), the PD function had a positive slope (Fig. $2 B)(E=1.5)$. With a shorter value of $\tau_{\text {syn }}(1 \mathrm{msec}$; red circles), the PD function had a negative slope similar to that achieved with a lower input intensity $\left(\tau_{\text {syn }}=2 \mathrm{msec}\right.$; $E=0.67$; black diamonds).

Although further reduction of input intensity (to $E=0.32$, $\tau_{\text {syn }}=2 \mathrm{msec}$ ) (Fig. $3 A$, black squares) caused an approximately proportional downward shift of the PD function along the $y$-axis, further shortening of $\tau_{\text {syn }}$ (to 0.5 and $0.25 \mathrm{msec}$; $=1.3$; red triangles and squares) both shifted down the function and changed its shape. The early part became steeper and the tail became flatter. This pattern reflected increased sensitivity at small phase delays, with less distinction among larger phase delays. In contrast, lengthening $\tau_{\text {syn }}$ from the control value (to 4 msec; red diamonds) shifted up the PD function, which decreased the relative difference in firing rates between phase delays.

We found that $\tau_{\text {syn }}$ controlled the position of the neuron along a continuum of operating modes (Bernander et al., 1991; Kisley and Gerstein, 1999; Rudolph and Destexhe, 2003): with very short versus very long values of $\tau_{\text {syn }}$, firing rate represented either input phase delay or input intensity, respectively. Thus with shorter versus longer $\tau_{\text {syn }}$, neurons were better coincidence detectors or classical temporal integrators, respectively. This principle is illustrated in Figure 3, $B$ and $C$.

Figure $3 B$, in a plot of PD index versus input intensity, summarizes the responses of 12 neurons to six values of $\tau_{\text {syn }}$. Shortening $\tau_{\text {syn }}$ below 2 msec shifted up the PD index trend line. The greater $Y$-intercept indicated a more negative PD function slope 
at a given input intensity, i.e., more sensitive CD. The consequently greater $X$-intercept indicated that negative $\mathrm{PD}$ function slopes could be found at a wider range of input intensities, i.e., more reliable CD. In contrast, lengthening $\tau_{\text {syn }}$ above 2 msec compressed the data points toward the $x$-axis (PD index $=0.1 \pm 0.13$, $\tau_{\text {syn }}=2 \mathrm{msec}, E \leq 1.3, n=14$; PD index $=$ $0.0 \pm 0.06, \tau_{\text {syn }}=8 \mathrm{msec}, E \leq 1.3, n=7$; $p=0.053$; two-tail $t$ test assuming unequal variances), i.e., less sensitive CD.

Figure $3 C$ demonstrates the performance of neurons as temporal integrators. Average firing rate is plotted as a function of input intensity at long and short values of $\tau_{\text {syn }}$ ( 8 and $0.5 \mathrm{msec}$, respectively). Each data point represents the firing rate averaged between all six phase delays of a PD function. With the longer $\tau_{\text {syn }}$, the trend line has a steeper slope $\left(49.6, R^{2}=0.47\right.$, $\tau_{\text {syn }}=8 \mathrm{msec} ; 17.8, R^{2}=0.68, \tau_{\text {syn }}=0.5$ $\mathrm{msec}$ ), indicating that firing rate was more sensitive to input intensity. Also, with the longer $\tau_{\text {syn }}$, the SD error bars are shorter $\left[3.46 \pm 0.477(\right.$ mean \pm SEM $), \tau_{\text {syn }}=8$ msec, $E \leq 1.3 ; 6.93 \pm 1.02, \tau_{\text {syn }}=0.5 \mathrm{msec}$, $E \leq 1.3 ; p=0.010$; two-tail $t$ test assuming unequal variances), indicating that firing rate varied less between phase delays, i.e., firing rate more reliably represented input intensity.

At any given value of $\tau_{\text {syn }}$, the PD function was somewhat influenced by input intensity, as evidenced by the negative slope of the trend lines in Figure $3 B$; however, sometimes an increase in input intensity could be compensated almost perfectly by a decrease in $\tau_{\text {syn }}$, as in Figure $3 A$ (red circles and black triangles). Four examples are illustrated in Figure 3D. This plot includes neurons in which PD functions at two intensity levels (data points connected by lines) were similar both in slope and $Y$-intercept. In all such cases, PD index was near zero, so the firing rate remained constant in the face of changes in both input intensity and phase delay.

The condition illustrated in Figure $3 D$ might occur when an independent increase in proximal synaptic noise accompanies an increase in synaptic signal intensity. Proximal synaptic noise would be mediated to a large extent by inhibitory input, which we investigated systematically in dynamic clamp experiments described below.

\section{Processes mediating the effects of $\boldsymbol{\tau}_{\text {syn }}$}

Figure 4 illustrates the processes that mediated the range of responses seen with different values of $\tau_{\text {syn. }}$. First, as anticipated, the width of PSPs decreased along with the width of PSCs (Fig. 4A). A narrower PSP resulted in a narrower integration time window, which made the PD function steeper. With a short $\tau_{\text {syn }}(0.5 \mathrm{msec})$, PSPs at a phase delay as small as $36^{\circ}$ rarely summated to threshold because of their rapid decay.

Second, PSP rise time exceeded PSC rise time, because $\tau_{\mathrm{m}}$ was longer than most $\tau_{\text {syn }}$ that we explored. When $\tau_{\text {syn }}$ was shortened, PSP rise was halted earlier, because the PSC peak arrived earlier. The curtailed rise reduced peak PSP amplitude. This accounts for
B
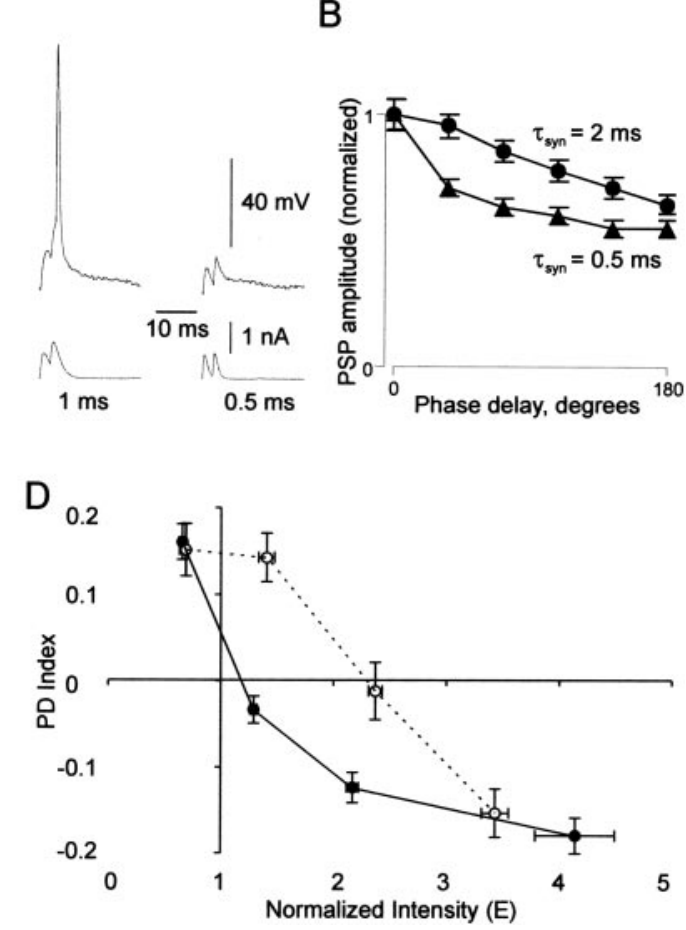

5

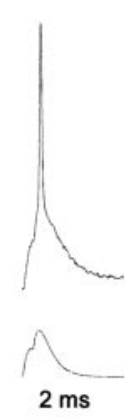

Normalized Intensity $(E)$

Figure 4. Properties mediating the effects of $\tau_{\text {syn }} \cdot A$, Traces of current injection and membrane voltage response in one neuron at $36^{\circ}$ phase delay with five different values of $\tau_{\text {syn }}$. $B$, Plots of peak PSP amplitude (mean \pm SE) versus phase delay in six neurons $\tau_{\text {syn }}=0.5 \mathrm{msec}$ (triangles) and $2 \mathrm{msec}$ (circles). Each phase delay is the average peak amplitude of all subthreshold PSPs in input intensity in current-clamp mode (black line) and dynamic clamp mode (dotted line). Each data point represents PD index Sersus $E$ (mean $\pm \mathrm{SE}$ ) for a group of data points (the current-clamp plot was calculated from the data set in Fig. 2D). $\mathrm{kNp} / T), k$ was calculated as $g(V-V)$ where $g$ is the unitary peak conductance used for dynamic clamp $V$ is the resting potential of the cell, and $V_{\mathrm{r}}$ is the reversal potential (always set at $0 \mathrm{mV}$ ) of the simulated synaptic conductance.

the downward shift of the PD function along the $y$-axis (Fig. $3 A$ ), an effect similar to that seen in Figure $2 B$ when input intensity was reduced (from $E=0.7$ to 0.5 ).

The second effect could be specific to the in vitro preparation. $\tau_{\mathrm{m}}$ remained constant and longer than $\tau_{\text {syn }}$ because of the constant and low level of synaptic input at or near the soma. Additional synaptic activity, as would be expected in vivo, would increase conductance and decrease $\tau_{\mathrm{m}}$; however, that decrease in $\tau_{\mathrm{m}}$ may itself compensate somewhat for the associated increase in input intensity. This hypothesis is supported by Figure $4 D$, a plot of PD index versus input intensity from an experiment in which excitatory inputs were delivered in dynamic clamp mode, which simulates natural synaptic conductance (Robinson and Kawai, 1993; Sharp et al., 1993). The data obtained in current-clamp mode (i.e., mean $\pm \mathrm{SE}$ of the data from Fig. $2 D$ ) are also plotted in Figure $4 D$ for comparison. Note that PD index values were higher in dynamic clamp mode (at least at intermediate intensity levels), indicating that the effective decrease in $\tau_{\mathrm{m}}$ attributable to the simulated synaptic conductance increased CD sensitivity.

In a third process, intrinsic somatic membrane conductances were responsible for the flat tail of the PD function seen with very short $\tau_{\text {syn }}$. A nonlinear process can be inferred from the biphasic shape of the PSP decay visible with the shortest $\tau_{\text {syn }}$ (Fig. $\left.4 A\right)\left(\tau_{\text {syn }}\right.$ $=0.5$ ). As a result of this biphasic decay, temporal summation of PSPs occurred in a biphasic pattern. We quantified this process in 
four neurons in Figure $4 B$. This is a plot of average subthreshold PSP peak amplitude at six phase delays, comparing the control $\tau_{\text {syn }}(2 \mathrm{msec})$ with short $\tau_{\text {syn }}(0.5 \mathrm{msec})$. With the control $\tau_{\text {syn }}$, peak amplitude declined smoothly with phase delay. With the short $\tau_{\text {syn }}$, peak amplitude dropped steeply initially and then more gradually in the last five phase delays, suggesting the presence of a persistent net depolarizing conductance, such as the persistent sodium current (Stafstrom et al., 1982). By slowing PSP decay, this conductance increased temporal summation of PSP tails, maintaining a non-zero firing rate in the later phase delays (Fig. $3 A$, red triangles and red squares).

Finally, intrinsic conductances were also responsible for repetitive firing, which contributed to the constant PD functions seen with long $\tau_{\text {syn }}$. Multiple spikes were generated during broad PSP peaks so the firing rate was no longer limited to $40 \mathrm{~Hz}$ at $0^{\circ}$ phase delay. Repetitive firing during broad PSP peaks is demonstrated in Figure $4 A$ ( $36^{\circ}$ phase delay).

\section{Inhibitory conductance increases $\mathrm{CD}$ sensitivity and reliability}

Another mechanism that might compensate for the influence of input intensity on the PD function is synaptic inhibition. The $\mathrm{GABA}_{\mathrm{A}}$ receptor-mediated conductance dominates synaptic input to the soma and proximal dendrites (Beaulieu et al., 1992; White et al., 1994), making it well suited for overcoming limitations imposed by somatic membrane properties.

In cerebellum (Hausser and Clark, 1997), auditory brainstem neurons (Funabiki et al., 1998), and hippocampal pyramidal neurons (Pouille and Scanziani, 2001), GABAergic synaptic inhibition has been found to increase sensitivity to input timing by narrowing the integration time window. This effect is mediated primarily by the added shunting conductance, similar to the effect of shortening $\tau_{\text {syn }}$. In addition, in auditory brainstem coincidence detectors, inhibition has been proposed to reduce sensitivity to input intensity (Pena et al., 1996; Yang et al., 1999). In that brain region, tolerance to input intensity may be built in by anatomical design through collateral excitation of inhibitory interneurons, so that inhibition is upwardly scaled with excitatory input intensity. In neocortex, collateral connections might allow similar upward scaling of inhibition with excitation (Galaretta and Hestrin, 1998; Varela et al., 1999).

To study the effect of somatic synaptic inhibition on the PD function, we simulated random inhibitory conductance in our input current trains using the dynamic clamp technique. Figure $5 A$ shows firing rate versus phase delay for the same neuron as in Figure $3 A$. As with shortened $\tau_{\text {syn }}$, the addition of $G_{\text {inh }}$ compensated for increased input intensity, changing a positive PD function slope to a negative one (compare filled circles with open circles); However, it affected the shape of the PD function differently. The contrasting effects are illustrated in Figure $5 B$, which shows negatively sloped PD functions (matched for firing rate at $0^{\circ}$ phase delay) averaged separately under the different conditions (for details, see Figure 5 legend). Under control conditions at low input intensity ( $E=0.6 \pm 0.06$; filled circles $)$, a smoothly sloped PD function was found. With short $\tau_{\text {syn }}(\leq 1 \mathrm{msec})$ at nearly twice the input intensity $(E=1.1 \pm 0.12$; X symbols), firing rate dropped rapidly at $36^{\circ}$ to nearly half its original value and then declined slowly. In contrast, in the presence of $G_{\text {inh }}$ with the same input intensity as with $\tau_{\text {syn }} \leq 1 \mathrm{msec}(E=1.1 \pm 0.09$; open circles), the PD function had a smooth slope similar to control conditions but was steeper, dropping to near 0 at $180^{\circ}$ phase delay.
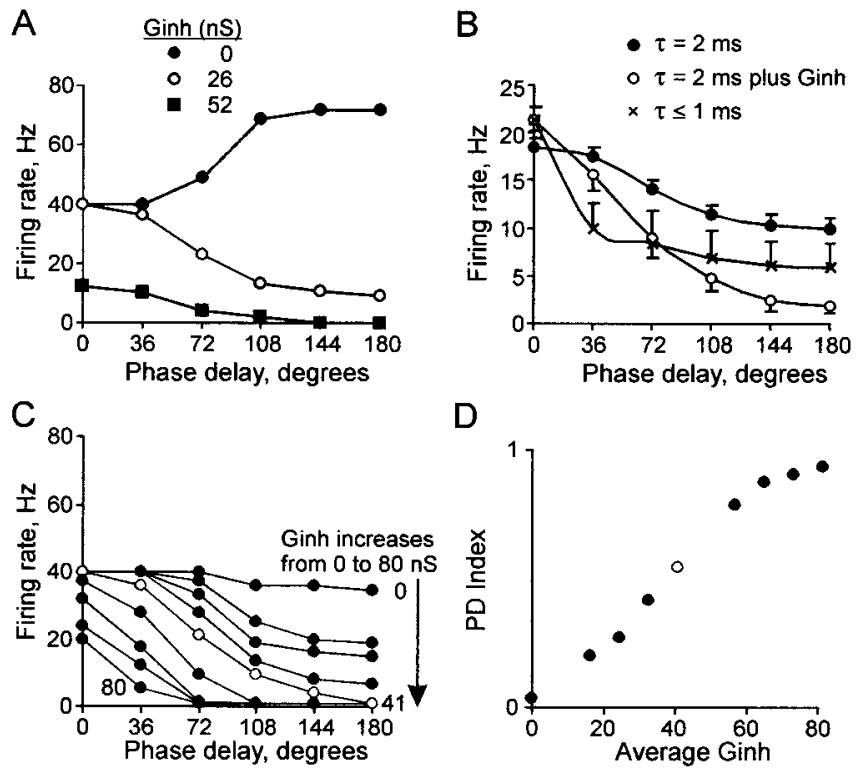

Figure 5. The effects of $G_{\text {inh. }} A$, Plots of firing rate versus phase delay in the absence (black circles) and presence of 26 and $52 \mathrm{nS} G_{\text {inh }}$ (open circles and squares, respectively), in the same neuron as Figure $3 A$. Input intensity was the same $(E=1.3)$ with all values of $G_{\text {inh. }} B$, Average of all PD functions with firing rates between 15 and $25 \mathrm{~Hz}$ at the $0^{\circ}$ phase delay, under control conditions (black circles): $n=12, E=0.6 \pm 0.06, P D$ index $=0.2 \pm 0.03$; with $G_{\text {inh }}$ (open circles): $\mathrm{n}=10, E=1.1 \pm 0.09, \mathrm{PD}$ index $=0.6 \pm 0.07$, average total $G_{\mathrm{inh}}=50 \mathrm{nS}$; and with $\tau_{\text {syn }} \leq 1 \mathrm{msec}(X$ symbols): $n=7, E=1.1 \pm 0.12, \mathrm{PD}$ index $=0.4 \pm 0.11$. C, Firing rate versus phase delay in one neuron, with stepwise addition of nine levels of $G_{\text {inh }}$ from 0 to $80 \mathrm{nS}$. The broadest range of firing rates between $0^{\circ}$ and $180^{\circ}$ phase delays is found with $G_{\text {inh }}=41 \mathrm{nS}$ (open circles). D,PD index versus $G_{\text {inh }} / E$ for the nine PD functions in C (open circle $=41 \mathrm{nS}$, as in C).

The stepwise addition of $G_{\text {inh }}$ resulted in a systematic change in the shape of the PD function (Fig. $5 C$, one representative neuron). The slope became more negative as $G_{\text {inh }}$ increased. The broadest range of firing rates between $0^{\circ}$ and $180^{\circ}$ was found in an intermediate range of average total $G_{\text {inh }}$ ( $41 \mathrm{nS}$; open symbols). With very high $G_{\text {inh }}(>50 \mathrm{nS})$, firing at the later phase delays was completely suppressed, resulting in a nearly binary distinction between synchronous and asynchronous input. The response of the neuron to the stepwise addition of $G_{\text {inh }}$ is illustrated in Figure $5 D$ by a steady rise in PD index.

PD index increased as a function of the inhibition to excitation ratio $\left(G_{\text {inh }} / E\right)$. This relationship can be seen in Figure $6 A$, which summarizes the responses of all 15 neurons in which $G_{\text {inh }}$ was used. In the plot, each PD index value in the presence of $G_{\text {inh }}$ (red dots) is aligned vertically above the value obtained at the same excitatory intensity in the absence of $G_{\text {inh }}$ (black dots).

A negatively sloped PD function could be produced at any input intensity with the addition of sufficient $G_{\text {inh. }}$. Figure $6 B$ summarizes the responses of 15 neurons in the presence of various levels of $G_{\text {inh }} / E$, in a plot of PD index versus input intensity. Addition of $G_{\text {inh }}$ shifted up the trend line (i.e., CD became more sensitive). As a result, the $X$-intercept increased beyond the highest intensities tested ( $E=2.4$ ) (i.e., CD became highly reliable).

Even with a constant level of $G_{\text {inh }} / E$, the PD function was somewhat influenced by input intensity, as evidenced by the negative slopes of the trend lines in Figure $6 B$; however, sometimes a particular negatively sloped PD function could be maintained in the face of increased input intensity by upward scaling of $G_{\text {inh }} / E$. Examples are shown in Figure $6 C$, a plot like that in Figure $3 D$ in 
which PD functions at two intensity levels (data points connected by lines) were similar in both slope and $Y$-intercept. In these cases, upward scaling of $G_{\text {inh }} / E$ perfectly compensated for the influence of input intensity on firing rate, making CD consistent across intensities. The low values of inhibition at which we observed these effects are within the range of inhibitory conductance seen during modest visual stimulation in vivo (i.e., $\sim 25 \mathrm{nS}$ ) (BorgGraham et al., 1998; Anderson et al., 2000). Theoretical considerations, however, imply that the larger values of inhibition used in this study might be attained during more intense activity (Beaulieu et al., 1992; Salin and Prince, 1996; Galarreta and Hestrin, 1997; Destexhe and Pare, 1999). GABA $A_{A}$ conductances of 70-90 nS have been reported in pyramidal cells in vitro in response to local extracellular stimulation (Connors et al., 1988) and in vivo during highly synchronized activity (e.g., during seizures) (Timofeev et al., 2002).

Processes that mediate the effects of $G_{\text {inh }}$ We identified three processes mediating the effects of $G_{\text {inh }}$. Figure $7 A$, showing current and $V_{\mathrm{m}}$ traces, demonstrates these effects in one neuron. The driving force of $G_{\text {inh }}$ on $V_{\mathrm{m}}$ toward its reversal potential $\left(E_{\mathrm{rev}}\right)$ is evident in the current trace. A brief outward current occurred during the action potential.

The first mediating process was a hyperpolarizing influence of $G_{\mathrm{inh}}$ on $V_{\mathrm{m}}$. This is illustrated by Figure $7 B$, in which individual peaks from traces in the presence and absence of $G_{\text {inh }}$ are overlaid. $G_{\text {inh }}$ reduced the amplitude of PSCs (bottom left) because of the increase in outward current with depolarization. This resulted in lower amplitude PSPs (top left). This hyperpolarizing effect caused a larger reduction of firing rate as phase delay increased because the minimally suprathreshold EPSPs at longer phase delays were made subthreshold by a relatively smaller decrease in amplitude.

Second, $G_{\text {inh }}$ shortened the time course of PSCs and consequently of PSPs, with faster rise and decay (Fig. $7 B$, center; both current and $V_{\mathrm{m}}$ scaled to height). This occurred because the increase in conductance shortened the effective membrane time constant. As seen with shortened $\tau_{\text {syn }}$, a shorter PSP narrowed the integration time window, which increased sensitivity to input phase delay. In contrast to the shortened $\tau_{\text {syn }}$, the increase in sensitivity with $G_{i n h}$ was not limited to the smallest phase delays by a flat PSP decay tail, because $V_{\mathrm{m}}$ was controlled primarily by the simulated GABAergic conductance instead of by intrinsic conductances. no $G_{\text {inh }}$ (filled circles).

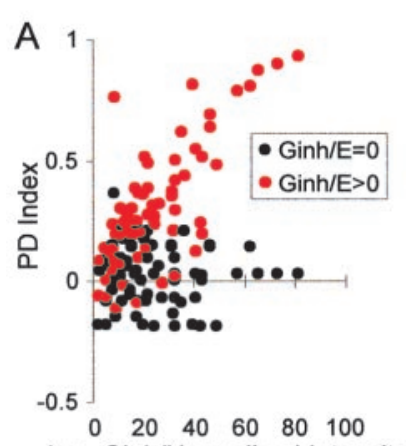

Avg. Ginh/Normalized Intensity $\mathrm{nS} / \mathrm{E}$

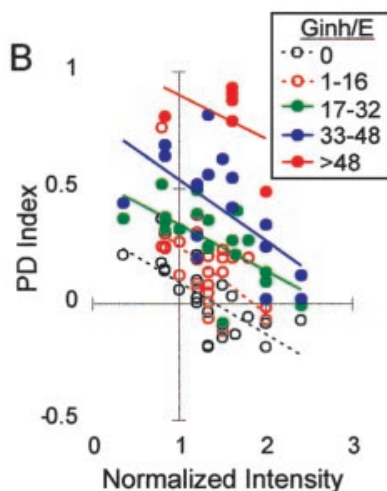

(E)

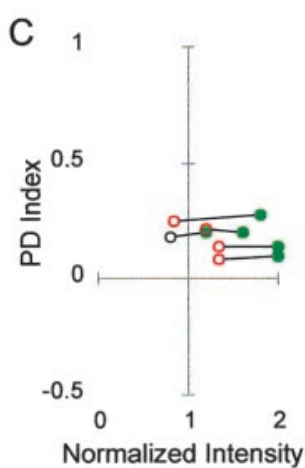

(E)
Figure 6. Effect of increasing $G_{\text {inh }} / E$ on a population of neurons. $A, P D$ index versus $G_{\text {inh }} / E$ in 15 neurons. $B, P D$ index versus input intensity at different values of $G_{\text {inh }} ; n=15$ neurons. $C$, Five examples in which both the slope and $Y$-intercept of the PD function were maintained in a neuron by upward scaling of $G_{\text {inh }} / E$ with intensity. For the two PD functions within a neuron (connected by lines), both the PD index and firing rate (average of all 6 phase delays) differed by $<10 \%$. The symbols for $G_{\text {inh }} / E$ are the same as in Figure $6 B$.
A

B
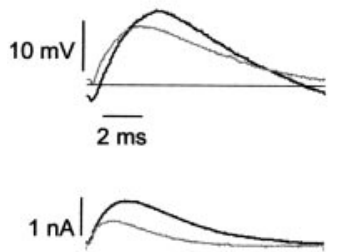

$180^{\circ}$ $0^{\circ}$
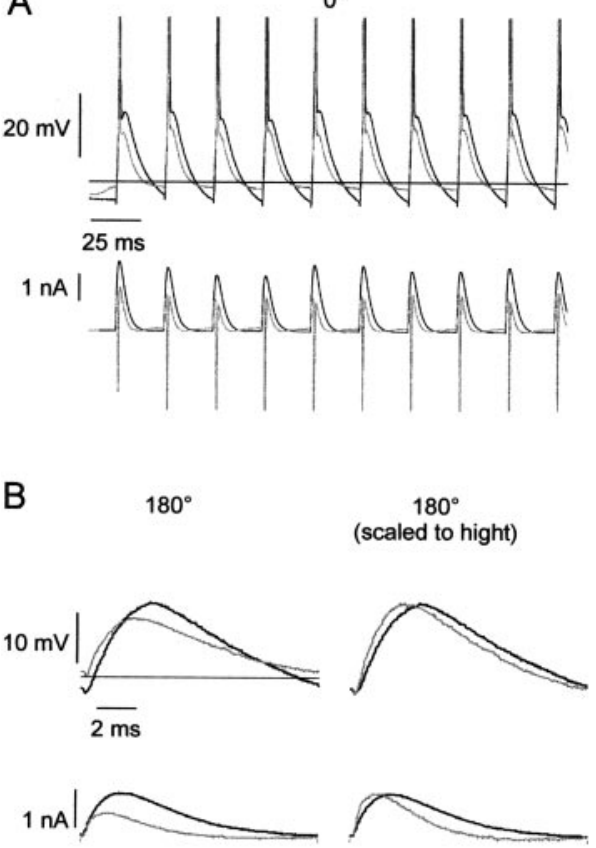
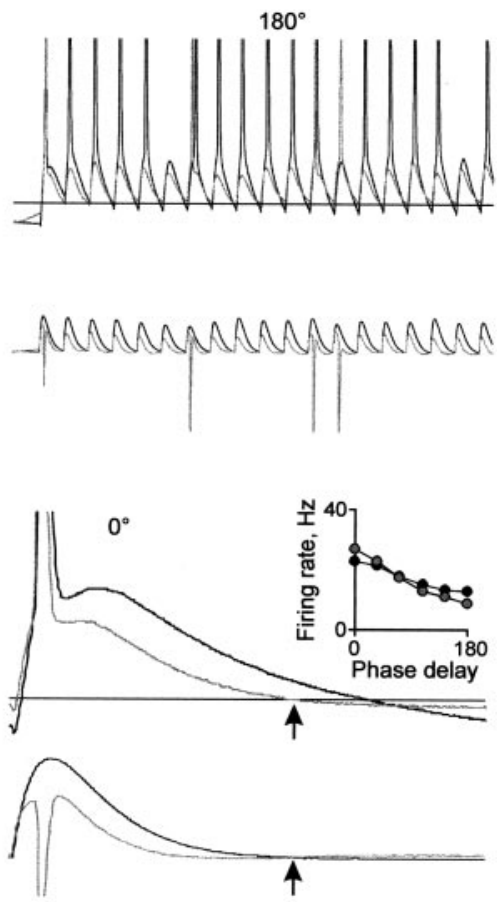

Figure 7. Processes mediating the effects of $G_{\text {inh. }} A$, Input current and $V_{\mathrm{m}}$ response in the absence (black) and presence (gray) of $G_{\text {inh }}$, at $0^{\circ}$ (left) and $180^{\circ}$ (right) phase delays. The horizontal line is $E_{\text {rev }}$ for $G_{\text {inh }}(-66 \mathrm{mV})$. $B$, Input current and $V_{\mathrm{m}}$ response for one stimulus cycle. Left, Subthreshold response from Figure $7 A$ (right) in the absence (black) and presence (gray) of $G_{\text {inh }}$; average PSC amplitude reduction for the subthreshold responses during four trains: $46.6 \%$. Center, Same traces as left, scaled to height, showing shortened membrane time constant attributable to $G_{\text {inh }}$. Right, The depolarizing force on $V_{\mathrm{m}}$ below $E_{\text {rev }}$ of $G_{\text {inh }}$. The current begins to increase after $V_{\mathrm{m}}$ falls below $E_{\text {rev }}$ (arrow). Inset, Firing rate versus phase delay for a different neuron. In the presence of $G_{\text {inh }}$ (gray circles), firing rate was boosted at the low phase delays and reduced at the high phase delays compared with
The third process was a depolarizing influence of $G_{\mathrm{inh}}$ on $V_{\mathrm{m}}$ (Gulledge and Stuart, 2003). This occurred during excursions negative to $E_{\text {rev }}$, such as an action potential afterhyperpolarization (Fig. $7 B$, right). As $V_{\mathrm{m}}$ fell below $E_{\text {rev }}$ (top right, arrow), the driving force of $G_{\text {inh }}$ reversed direction. The reversal is evident in the upward deflection in the current (bottom right, arrow). The depolarizing influence appeared to be most significant at the lower phase delays, as seen by the subtle boosting of the firing rate 
A

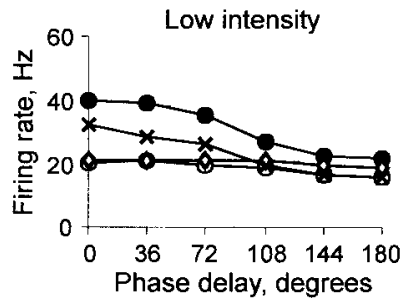

B
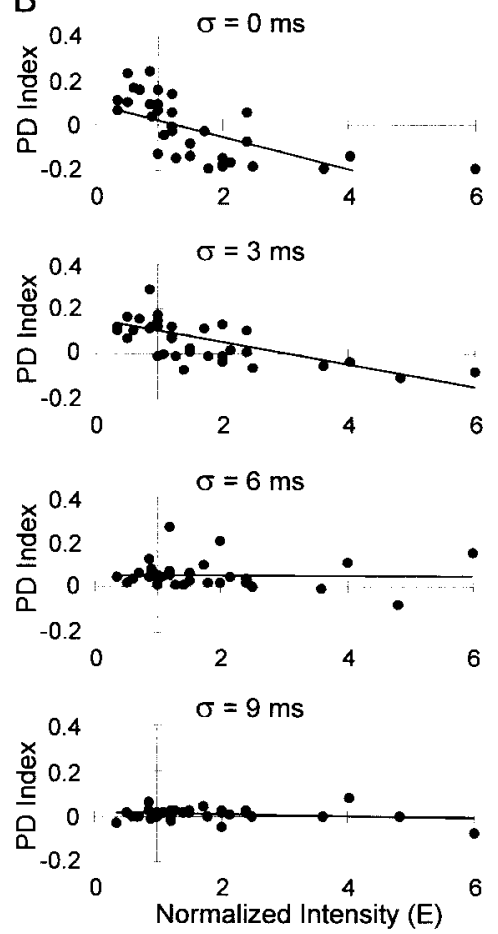

Figure 8. Effects of input jitter. $A$, Firing rate versus phase delay with $\sigma=0,3,6$, and 9 msec of jitter, at low input intensity ( $E=1$, left) and high input intensity ( $E=2$, right). $B$, PD index versus input intensity at each jitter level $(\sigma=0,3,6$, and 9 msec; top to bottom) in 12 neurons. $C$, Examples of input current (insets) and corresponding interspike interval histogram for each jitter level ( $\sigma=0,3,6$, and 9 msec; top to bottom).

at the lower phase delays (Fig. $7 B$, inset). This was attributable to the greater elapsed time between peaks during which $V_{\mathrm{m}}$ remained below $E_{\text {rev }}$ (Fig. $7 A$, compare left and right panels). In vivo, the depolarizing effect would be greatest during periods of slow firing and for neurons with large afterhyperpolarizations that go below $E_{\mathrm{rev}}$. If $E_{\mathrm{rev}}$ were significantly more negative (e.g., approximately $-75 \mathrm{mV}$ ), as has been suggested by some in vivo intracellular studies in adult cortex (Pare et al., 1998), the depolarizing effect would become less significant.

Jitter lengthens the time course and reduces the amplitude of current fluctuations

The precision of correlated firing of neurons varies widely in different areas of cortex and under different behavioral conditions. Peak width of cross-correlations of spike activity ranges from several to tens of milliseconds (Castelo-Branco et al., 2000; Baker et al., 2001). Variation in the arrival time of synchronized synaptic events (jitter) would influence $V_{\mathrm{m}}$ at the soma. We explored the influence of jitter on the PD function at different input intensity levels.

In a typical neuron, a large jitter $(\sigma=9 \mathrm{msec})$ obscured input phase delay, resulting in a constant PD function at both low- and high-input intensity levels (Fig. $8 \mathrm{~A}$, left and right, diamonds). A small jitter ( $\sigma=3-6 \mathrm{msec})$, however, could compensate some- what for increased input intensity. When input intensity was low, it caused a downward shift (Fig. $8 \mathrm{~A}$, left, from filled circles to $\mathrm{X}$ symbols) and some flattening. When input intensity was high, it could transform a positive into a slightly negative slope (Fig. 8 A, right, from filled circles to open circles).

The effect of input jitter on 12 neurons is summarized in the plots of PD index versus input intensity at each level of jitter (Fig. $8 B$ ). The $\sigma=0$ plot is a subset of Figure $2 D$. As jitter was increased from $\sigma=0-6 \mathrm{msec}$, the slope of the trend line became less negative (i.e., CD became more consistent across input intensities); however, at $\sigma=9 \mathrm{msec}$, PD index values at all intensities were close to zero. Thus, as $\sigma$ increased there was a decrease in and eventual loss of CD sensitivity.

The current traces in Figure $8 C$ illustrate how jitter produced these effects. Interspike interval histograms are shown below each current waveform. When inputs were jittered, the reduced synchrony between neurons within each group resulted in current peaks that were both broader and lower amplitude. The broadening of current peaks lengthened the integration time window similar to the effect of a long $\tau_{\text {syn }}$, blurring the distinction between phase delays, thereby making the PD function more nearly constant. Peak broadening was the primary effect with $\sigma=9 \mathrm{msec}$, where current fluctuations resembled noise. A small jitter $(\sigma=3$ or $6 \mathrm{msec})$, however, reduced current amplitude without abolishing periodic input structure, so firing rate was reduced preferentially at greater phase delays, as with decreased input intensity.

The combination of both jitter and $G_{\text {inh }}$ enhances sensitivity and consistency

Synaptic input to neurons in vivo contains complex combinations of the influences that we have explored. To investigate how multiple influences might interact to alter the PD function, we used input trains with both $G_{\text {inh }}$ and jitter.

As described above, adding $G_{\text {inh }}$ increased CD sensitivity (increasing the $Y$-intercept of trend line) (Fig. $6 B$ ) but not its consistency across input intensities (negatively sloped trend lines) (Fig. 6B). In contrast, jitter decreased CD sensitivity but increased its consistency (trend line slope approaching zero) (Fig. $8 B)$. We found that with both jitter and a constant level of $G_{\text {inh }}$ (no supralinear increase in $G_{\text {inh }} / E$ required), firing rate was consistently sensitive to input phase delay regardless of input intensity.

The combined effects can be seen by comparing three levels of jitter on the plot of PD index versus input intensity: $\sigma=0,3$, and $6 \mathrm{msec}$ (Figs. $6 B, 9 A, B$ ). Each plot includes a range of $G_{\text {inh }}$ levels. As when jitter was added alone (compare Fig. $6 B$ with Fig. 9, $A$ or $B$ ), the band of data points for a given level of $G_{\text {inh }}$ (e.g., $G_{\text {inh }} / E=$ 1-16; open red circles) tended to slope downward less steeply, indicating more consistent CD across input intensities. The ad- 

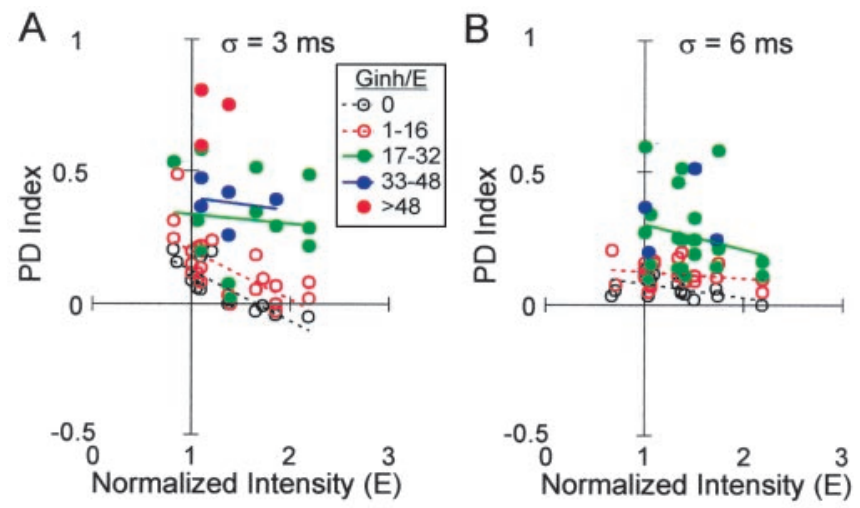

Figure 9. Effect of combined $G_{\text {inh }}$ and input jitter. $A, P D$ index versus input intensity with $\sigma=3 \mathrm{msec}$, at different levels of $G_{\text {inh }}$., PD index versus input intensity with $\sigma=6 \mathrm{msec}$, at different levels of $G_{\text {inh. }}$. There is an insufficient number of data points for the highest $G_{\text {inh }}$ in both plots to show a trend line.

dition of $G_{\text {inh }}$, however, increased CD sensitivity without loss of consistency across input intensities (e.g., for $\sigma=6 \mathrm{msec}$ ) (compare Figs. $8 B, 9 B)$.

\section{Discussion}

We investigated whether neocortical pyramidal neurons could perform a form of CD by exploring their firing rate representation of input phase delay. Under control conditions, input intensity determined whether firing rate increased or decreased with greater phase delay or was independent of phase delay (Figs. $2 \mathrm{~B}-$ $D)$. These findings in rat cortical neurons were consistent with those from computer simulations of a different form of CD (Bernander and Koch, 1994; Murthy and Fetz, 1994; Rudolph and Destexhe, 2001) in which input timing was represented by the distribution of events within a single input group, rather than by the phase delay between two input groups. We extended these findings by showing that when input features were altered, the influence of input intensity decreased. The input train features that we altered represented dynamic properties of synaptic input that vary from moment to moment in vivo.

By shortening $\tau_{\text {syn }}$, we simulated an increase in dendritic membrane conductance, as might occur in vivo during periods of increased synaptic noise (Destexhe and Pare, 1999). Shortening $\tau_{\text {syn }}$ could compensate for the influence of increased input signal intensity, switching the PD function slope from positive to negative (Fig. 3A). This finding suggests that the increase in conductance resulting from noisy background activity in vivo could help maintain reliable $C D$ at a wide range of input signal intensities (Fig. 3B). As expected (Bernander et al., 1991; Kisley and Gerstein, 1999; Rudolph and Destexhe, 2003), we found that $\tau_{\text {syn }}$ controlled the position of the neuron along a continuum of operating modes between CD and temporal integration: a short or long $\tau_{\text {syn }}$, respectively, made firing rate more sensitive to input phase delay or input intensity (Fig. $3 C, D$ ). Shortening $\tau_{\text {syn }}$ below $2 \mathrm{msec}$ preferentially increased the sensitivity of firing rate to small $\left(<36^{\circ}\right)$ input phase delays. This effect was mediated in part by intrinsic membrane conductances that endowed the PD function with a distinctive biphasic shape (Fig. $4 B$ ).

Adding simulated inhibitory conductance shortened the effective $\tau_{\mathrm{m}}$. The addition of sufficient $G_{\text {inh }}$ could compensate for the influence of increased input intensity, either switching the PD function slope from positive to negative or shifting down the PD function (Fig. 5A). This finding suggests that collateral activation of inhibitory interneurons in vivo could help maintain CD reliability at a wide range of input intensities (Fig. 6B). We found that $\mathrm{CD}$ sensitivity increased with $G_{\text {inh }} / E$ (Fig. $6 A$ ). Supralinear scaling of inhibition with excitation, attributable to differential activity-dependent plasticity at inhibitory and excitatory synapses, has been proposed to maintain circuit stability and underlie sensory contrast adaptation in cortex (Galaretta and Hestrin, 1998; Varela et al., 1999). Our results suggest that an additional role for such supralinear scaling may be to increase CD consistency (Fig. $6 C)$. We also found that different values of $G_{\text {inh }} / E$ altered the character of CD in distinctly different ways, from a smooth gradation of firing rate as a function of phase delay to a nearly binary distinction between small $\left(<36^{\circ}\right)$ and all other phase delays (Fig. $5 C$ ).

A large jitter abolished CD; however, a small jitter could compensate for increased input intensity, either switching the slope of the PD function from positive to negative or decreasing its $Y$-intercept (Fig. $8 \mathrm{~A}$ ). A moderate level of jitter combined with a constant $G_{\text {inh }}$ produced CD that was somewhat sensitive and also consistent across a wide range of input intensities (Fig. 9B, open red circles).

\section{Context for the study}

The effect of input timing on neuronal output was addressed in several recent studies (Funabiki et al., 1998; Pouille and Scanziani, 2001; Williams and Stuart, 2002) by investigating the time window within which two marginally subthreshold inputs could evoke an action potential. In that approach, concerned with the efficacy of synaptic inputs, the output feature of interest was the probability of producing an output spike. In contrast, the output feature of interest in our study was the firing rate of the neuron. To study the net influence of an input variable on the output of the neuron over time, we used input trains. The resulting ability to combine two suprathreshold inputs allowed us to explore the influence of input intensity. We did not address the question of whether some other output feature, such as precise spike timing, might be used to signal input timing.

Firing rate is of interest because of its known functional significance in some pyramidal neurons. For example, in primary motor cortex it has been found to correlate with parameters of movement such as force (Evarts, 1968) and direction (Georgopoulos et al., 1982). In this context, dynamic influences on firing rate may play an important role in motor control.

We used input phase delay as a systematic, computationally straightforward model of input timing, as it is used in studies of auditory brainstem neurons (Pena et al., 1996; Reyes et al., 1996; Cook et al., 2003). The unrealistically large voltage transients of our simulated inputs exaggerated the effects of temporal summation that would be expected in vivo while allowing exploration of underlying mechanisms. This study was limited to phase delay between inputs at cortical gamma oscillation frequency $(40 \mathrm{~Hz})$. At higher or lower frequencies, the degree of temporal summation between input groups would likely increase or decrease, resulting in different PD function slopes; however, the major effects of $\tau_{\text {syn }}, G_{\text {inh }}$, and jitter would not be expected to differ.

A computational role for input phase delay in neocortex has been proposed by Hopfield (1995). In his theory, the phase of an action potential with respect to a transient local oscillatory rhythm could represent an analog variable, such as a sensory input. Analog pattern recognition such as in sensory perception might occur when a neuron receives synaptic input simultaneously from many pathways with different delays. A simple example of such an operation with two incoming pathways underlies binaural sound localization in auditory brainstem coinci- 
dence detectors (Goldberg and Brown, 1969; Carr and Konishi, 1990). In hippocampal "place cells," the phase of synaptic input in relation to the local theta rhythm may represent the animal's spatial location (O'Keefe and Recce, 1993). Our study explores the viability of phase delay within the gamma oscillation frequency for computation in neocortex.

Neural synchrony, represented by the $0^{\circ}$ phase delay in our study, has increasingly been observed in neocortex in relation to behavior, for example in motor control (Vaadia et al., 1995; Riehle et al., 1997; Hatsopoulos et al., 1998). The degree of synchrony has been found to vary with behavioral state (Murthy and Fetz, 1996; Baker et al., 2001); however, synchrony has also been dismissed as a byproduct of neural processing rendered meaningless by the low timing sensitivity of pyramidal neurons (Shadlen and Movshon, 1999). Our findings suggest several mechanisms by which neocortex may compensate for these limitations. Although the biophysical properties of neocortical pyramidal cells may not optimize them for CD, their attributes and circuitry may support this task when needed.

\section{Implications for neural coding}

Our results are consistent with past evidence that input synchronization can efficiently increase firing rate (Segundo et al., 1963; Reyes, 2003); however, as input intensity increases, its efficiency decreases. When input intensity exceeds a threshold, synchronization instead causes the firing rate to fall. The intensity threshold is a boundary beyond which input synchronization may be viewed as a means of gain control, which could be useful for allowing a neuron to ignore redundancy or excessive input.

We have shown several mechanisms that can be used to shift the boundary, to extend the intensity range over which synchronization either efficiently increases firing rate or else acts as gain control. We have also described mechanisms that reduce the responsiveness of the neuron to input synchronization. The firing rate response to synchronized inputs may also be shaped by other dynamic factors such as inhibitory input timing (Gauck and Jaeger, 2000; Galarreta and Hestrin 2001), synaptic depression (Abbott et al., 1997; Kuba et al., 2002; Cook et al., 2003), and chemical neuromodulators.

Each of the three relationships between input synchrony and firing rate may be useful for several different computational tasks. First, a positive correlation is used by auditory brainstem coincidence detectors, which adjust their firing rate to reflect interaural time difference, a continuous parameter that is used to compute sound location. This operation (found at low intensities) with a smoothly graded PD function (Fig. 5C, open circles) may be used in motor cortex during tasks in which the firing rate of a corticospinal neuron controls a continuous parameter of movement such as force (Evarts, 1968). This would be consistent with observations of Baker et al. (2001). During a precision grip task, the highest neural synchrony was observed during a steady hold period, when activity in motor cortex was at low intensity. Baker et al. (2001) noted that slow, synchronous motor cortex input to corticospinal neurons might allow for efficient recruitment when demands on cortical processing are low.

The precise shape of a decreasing PD function might be relevant to its computational use. For example, with a steeply decreasing function, highly synchronous inputs would be far more effective in generating action potentials than inputs with only a slight timing disparity. A steeply decreasing function might be useful in visual cortex for feature grouping, where a binary result is desirable (i.e., either the feature is part of the group or it is not). The narrow peaks found in cross-correlation analysis of spike times in visual cortex during these tasks (Roelfsema et al., 1994; Castelo-Branco et al., 2000) suggest that precise, discrete inputs required for a binary computation may be available.

The second relationship is the negative correlation between input synchrony and firing rate found at high intensities. In the precision grip task of Baker et al. (2001), the lowest neural synchrony was observed during precise movements, when activity in motor cortex was most intense. According to our findings, neurons receiving high intensity input could operate with an increasing PD function, such that an increase in their firing rate would be achieved only by desynchronizing existing inputs or by adding asynchronous inputs. This operation would control gain by ignoring redundant synchronous input.

The gain control function could also be useful in situations of large scale synchrony such as slow-wave sleep. Here it could protect against undesirable consequences of excessive firing such as glutamate toxicity or seizure activity. When seizures do occur, this same gain control may fortuitously provide damage control.

The third relationship between input phase delay and firing rate is independence. When a constant response function occurred because of a large jitter or a long $\tau_{\text {syn }}$, firing rate reflected input intensity reliably and sensitively. This classical "rate coding" strategy describes the operation performed by an orientation-selective neuron in the visual cortex (Hubel and Wiesel, 1962). The firing rate of this type of neuron is determined by the intensity of its thalamic input, which in turn is determined by the proportion of its receptive field stimulated by light. To enhance such a computation, spurious variations in input synchrony might be compensated by adjustments in conductance and jitter.

We have shown how neural coding in neocortex could be influenced by processes that vary on a behaviorally relevant time scale. We have also discussed how the coding strategy could be in flux with changes in behavioral state. The existence of multiple interpretations of firing rate in a constant state of flux would seem to leave the decoding target neuron with a complex problem. The firing rate of any single input to the decoding neuron would necessarily be ambiguous; however, the firing behavior of the decoding neuron itself is determined not by a signal from any one of its inputs, but instead by activity among its input population. For example, if the neuron in Figure $2 B$ represented the corticospinal neuron of Baker et al. (2001), it could be instructed to drive a muscle (via a target motor neuron) to produce about the same force when receiving either low intensity synchronous input $(E=$ $0.5 ; 0^{\circ}$ phase delay) or somewhat higher intensity asynchronous input ( $E=0.7 ; 108^{\circ}$ phase delay). To function successfully, the decoding target motor neuron need not unambiguously interpret how synchronous was the input to the corticospinal neuron.

Our study has introduced the notion of a varying intensity boundary at which the effect of input synchrony on the firing rate of a neuron is determined. Below this boundary, synchrony increases firing rate, whereas above the boundary, synchrony limits firing rate. In each neuron, this boundary may be shifted by dynamic influences that vary with behavioral state.

\section{References}

Abbott LF, Varela JA, Sen K, Nelson SB (1997) Synaptic depression and cortical gain control. Science 275:220-224.

Abeles M (1991) Corticonics: neural circuits of the cerebral cortex. Cambridge, MA: Cambridge UP. 
Anderson JS, Carandini M, Ferster D (2000) Orientation tuning of input conductance, excitation, and inhibition in cat primary visual cortex. J Neurophysiol 84:909-926.

Baker SN, Spinks R, Jackson A, Lemon RN (2001) Synchronization in monkey motor cortex during a precision grip task. I. Task-dependent modulation in single-unit synchrony. J Neurophysiol 85:869-885.

Beaulieu C, Kisvarday Z, Somogyi P, Cynader M, Cowey A (1992) Quantitative distribution of GABA-immunopositive and -immunonegative neurons and synapses in the monkey striate cortex (area 17). Cereb Cortex 2:295-309.

Bernander O, Koch C (1994) The effect of synchronized inputs at the single neuron level. Neural Comput 6:622-641.

Bernander O, Douglas RJ, Martin KAC, Koch C (1991) Synaptic background activity influences spatiotemporal integration in single pyramidal cells. Proc Natl Acad Sci USA 88:11569-11573.

Borg-Graham LJ, Monier C, Fregnac Y (1998) Visual input evokes transient and strong shunting inhibition in visual cortical neurons. Nature 393:369-373.

Carr CE, Konishi M (1990) A circuit for detection of interaural time differences in the brain stem of the barn owl. J Neurosci 10:3227-3246.

Castelo-Branco M, Goebel R, Neuenschwander S, Singer W (2000) Neural synchrony correlates with surface segregation rules. Nature 405:685-689.

Connors BW, Gutnick MH (1990) Intrinsic firing patterns of diverse neocortical neurons. Trends Neurosci 13:99-103.

Connors BW, Malenka RC, Silva LR (1988) Two inhibitory postsynaptic potentials, and $\mathrm{GABA}_{\mathrm{A}}$ and $\mathrm{GABA}_{\mathrm{B}}$ receptor-mediated responses in neocortex of rat and cat. J Physiol (Lond) 406:443-468.

Cook DL, Schwindt PC, Grande LA, Spain WJ (2003) Synaptic depression in the localization of sound. Nature 421:66-70.

Destexhe A, Pare D (1999) Impact of network activity on the integrative properties of neocortical pyramidal neurons in vivo. J Neurophysiol 81:1531-1547.

Evarts EV (1968) Relation of pyramidal tract activity to force exerted during voluntary movement. J Neurophysiol 31:14-27.

Funabiki K, Koyano K, Ohmori H (1998) The role of GABAergic inputs for $\mathrm{CD}$ in the neurones of nucleus laminaris of the chick. J Physiol (Lond) 508:851-869.

Galarreta M, Hestrin S (1998) Frequency-dependent synaptic depression and the balance of excitation and inhibition in the neocortex. Nat Neurosci 1:587-594.

Galarreta M, Hestrin S (2001) Spike transmission and synchrony detection in networks of GABAergic interneurons. Science 292:2295-2299.

Gauck V, Jaeger D (2000) The control of rate and timing of spikes in the deep cerebellar nuclei by inhibition. J Neurosci 20:3006-3016.

Georgopoulos AP, Kalaska JF, Caminiti R, Massey JT (1982) On the relations between the direction of two-dimensional arm movements and cell discharge in primary motor cortex. J Neurosci 2:1527-1537.

Gerstner W, Kreiter AK, Markram H, Herz AVM (1997) Neural codes: firing rates and beyond. Proc Natl Acad Sci USA 94:12740-12741.

Goldberg JM, Brown PB (1969) Response of binaural neurons of dog superior olivary complex to dichotic tonal stimuli: some physiological mechanisms of sound localization. J Neurophysiol 32:613-636.

Gulledge AT, Stuart GJ (2003) Excitatory actions of GABA in the cortex. Neuron 37:299-309.

Harsch A, Robinson HPC (2000) Postsynaptic variability of firing in rat cortical neurons: the roles of input synchronization and synaptic NMDA receptor conductance. J Neurosci 20:6181-6192.

Hatsopoulos NG, Ojakangas CL, Paninski L, Donoghue JP (1998) Information about movement direction obtained from synchronous activity of motor cortical neurons. Proc Natl Acad Sci USA 95:15706-15711.

Hausser M, Clark BA (1997) Tonic synaptic inhibition modulates neuronal output pattern and spatiotemporal synaptic integration. Neuron 19:665-678.

Hausser M, Spruston N, Stuart GJ (2000) Diversity and dynamics of dendritic signaling. Science 290:739-744.

Ho N, Destexhe A (2000) Synaptic background activity enhances the responsiveness of neocortical pyramidal neurons. J Neurophysiol 84:1488-1496.

Hopfield JJ (1995) Pattern recognition computation using action potential timing for stimulus representation. Nature 376:33-36.

Hubel DH, Wiesel TN (1962) Receptive fields, binocular interaction and functional architecture in the cat's visual cortex. J Physiol (Lond) 160:106-154.

Kisley MA, Gerstein GL (1999) The continuum of operating modes for a passive model neuron. Neural Comput 11:1139-1154.

Koch C, Rapp M, Segev I (1996) A brief history of time (constants). Cereb Cortex 6:93-101.

Kuba H, Koyana K, Ohmori H (2002) Synaptic depression improves CD in the nucleus laminaris in brainstem slices of the chick embryo. Eur J Neurosci 15:984-990.

Mainen ZF, Sejnowski TJ (1995) Reliability of spike timing in neocortical neurons. Science 268:1503-1506.

Marsalek P, Koch C, Maunsell J (1997) On the relationship between synaptic input and spike output jitter in individual neurons. Proc Natl Acad Sci USA 94:735-740.

Murthy VN, Fetz EE (1994) Effects of input synchrony on the firing rate of a three-conductance cortical neuron model. Neural Comput 6:1111-1126.

Murthy VN, Fetz EE (1996) Oscillatory activity in sensorimotor cortex of awake monkeys: synchronization of local field potentials and relation to behavior. J Neurophysiol 76:3949-3967.

O’Keefe J, Recce ML (1993) Phase relationship between hippocampal place units and the EEG theta rhythm. Hippocampus 68:317-330.

Oviedo H, Reyes AD (2002) Boosting of neuronal firing evoked with asynchronous and synchronous inputs to the dendrite. Nat Neurosci 5:261-266

Pare D, Lang EJ, Destexhe A (1998) Inhibitory control of somatic and dendritic sodium spikes in neocortical pyramidal neurons in vivo: an intracellular and computational study. Neuroscience 84:377-402.

Pena JL, Viete S, Albeck Y, Konishi M (1996) Tolerance to sound intensity of binaural coincidence detection in the nucleus laminaris of the owl. J Neurosci 16:7046-7054.

Pouille F, Scanziani M (2001) Enforcement of temporal fidelity in pyramidal cells by somatic feed-forward inhibition. Science 293:1159-1163.

Rall W (1977) The nervous system. In: Handbook of physiology, Vol 1 (Kandel ER, ed), pp 39-97. Bethesda, MD: American Physiological Society.

Reyes AD (2003) Synchrony-dependent propagation of firing rate in iteratively constructed networks in vitro. Nat Neurosci 6:593-599.

Reyes AD, Fetz EE (1993) Two modes of interspike interval shortening by brief transient depolarizations in cat neocortical neurons. J Neurophysiol 69:1661-1672.

Reyes AD, Rubel EW, Spain WJ (1996) In vitro analysis of optimal stimuli for phase-locking and time-delayed modulation of firing in avian nucleus laminaris neurons. J Neurosci 16:993-1007.

Riehle A, Grun S, Diesmann M, Aertsen A (1997) Spike synchronization and rate modulation differentially involved in motor cortical function. Science 278:1950-1953.

Rieke F, Warland D, de Ruyter Van Steveninck, Bialek W (1996) Spikes: exploring the neural code. Cambridge, MA: MIT.

Robinson HPC, Kawai N (1993) Injection of digitally synthesized synaptic conductance transients to measure the integrative properties of neurons. J Neurosci Methods 49:157-165.

Roelfsema PR, Konig P, Engel AK, Sireteanu R, Singer W (1994) Reduced synchronization in the visual cortex of cats with strabismic amblyopia. Eur J Neurosci 6:1645-1655.

Rudolph M, Destexhe A (2001) Correlation detection and resonance in neural systems with distributed noise sources. Physiol Rev Lett 86:3662-3665.

Rudolph M, Destexhe A (2003) Tuning neocortical pyramidal neurons between integrators and coincidence detectors. J Comput Neurosci 14:239-251.

Salin PA, Prince DA（1996） Spontaneous GABAA receptor-mediated inhibitory currents in adult rat somatosensory cortex. J Neurophysiol 75:1573-1588.

Schwindt P, O’Brien JA, Crill W (1997) Quantitative analysis of firing properties of pyramidal neurons from layer 5 of rat sensorimotor cortex. J Neurophysiol 77:2484-2498.

Segundo JP, Moore GP, Stensaas LJ, Bullock TH (1963) Sensitivity of neurons in Aplysia to temporal pattern of arriving impulses. J Exp Biol 40:643-667.

Shadlen MN, Movshon JA (1999) Synchrony unbound: a critical evaluation of the temporal binding hypothesis. Neuron 24:67-77. 
Sharp AA, O'Neil MB, Abbott LF, Marder E (1993) The dynamic clamp: artificial conductances in biological neurons. Trends Neurosci 16:389-394.

Softky W (1994) Sub-millisecond CD in active dendritic trees. Neuroscience 58:13-41.

Stafstrom CE, Schwindt PC, Crill WE (1982) Negative slope conductance due to a persistent subthreshold sodium current in cat neocortical neurons in vitro. Brain Res 236:221-226.

Stuart G (1999) Voltage-activated sodium channels amplify inhibition in neocortical pyramidal neurons. Nat Neurosci 2:144-150.

Timofeev I, Grenier F, Steriade M (2002) The role of chloride-dependent inhibition and the activity of fast-spiking neurons during cortical spikewave electrographic seizures. Neuroscience 114:1115-1132.

Vaadia E, Haalman I, Abeles M, Bergman H, Prut Y, Slovin H, Aertsen A
(1995) Dynamics of neuronal interactions in monkey cortex in relation to behavioral events. Nature 373:515-518.

Varela JA, Son S, Turrigiano GG, Nelson SB (1999) Differential depression at excitatory and inhibitory synapses in visual cortex. J Neurosci 19:4293-4304.

White EL, Amitai Y, Gutnick MJ (1994) A comparison of synapses onto the somata of intrinsically bursting and regular spiking neurons in layer $\mathrm{V}$ of rat SmI cortex. J Comp Neurol 342:1-14.

Williams SR, Stuart GJ (2002) Dependence of EPSP efficacy on synapse location in neocortical pyramidal neurons. Science 295:1907-1910.

Yang L, Monsivais P, Rubel EW (1999) The superior olivary nucleus and its influence on nucleus laminaris: a source of inhibitory feedback for coincidence detection in the avian auditory brainstem. J Neurosci 19:2313-2325. 